\title{
Produção consorciada de tilápias e vegetais em sistema de aquaponia ${ }^{1}$
}

\author{
Gilvan Gonçalves Contini², Luciano Rodrigues Belo², Rodrigo Gomes Branquinho ${ }^{2}$
}

\begin{abstract}
Resumo: O sistema de aquaponia é pouco conhecido no Brasil, e consiste na integração dos cultivos de plantas e de peixes. O objetivo com o presente estudo foi avaliar o crescimento de tilápias criadas com reutilização de água e a adaptação de hortaliças ao sistema de aquaponia. Foi instalado um sistema de aquaponia com a criação de tilápias conjugada com a produção de agrião (Nasturtium officinale), alface (Lactuca sativa) e cebolinha (Allium fistulosum). Estas espécies vegetais foram também cultivadas em canteiros de terra. Os vegetais foram colhidos 30 dias após o plantio, sendo obtidas medidas de massa da matéria verde e seca. O crescimento das tilápias foi monitorado obtendo-se, semanalmente, medidas de peso e comprimento. Os resultados mostraram que a criação de peixes e o cultivo de vegetais podem ser conjugados em um único sistema, capaz de oferecer bons resultados. A aquaponia mostrou ser um ambiente mais vantajoso à produção de agrião e cebolinha, chegando a produzir mais de $75 \%$ quando comparado a canteiros de terra. Tilápias criadas em viveiros desse sistema podem alcançar taxa de crescimento diário de $0,06 \mathrm{~cm} \mathrm{dia}^{-1}$, desde que feito o arraçoamento correto.
\end{abstract}

Palavras chave: Tilápia; Agrião; Cebolinha; Alface; Aquaponia.

\section{Consortium production of tilapia and vegetables in aquaponics system}

\begin{abstract}
The aquaponics system is little known in Brazil, and consists of the integration of plant and fish crops. The aim of the present study was to evaluate the growth of tilapia raised with water reuse and the adaptation of vegetables to the aquaponics system. An aquaponics system was installed with the creation of tilapia combined with the production of watercress (Nasturtium officinale), lettuce (Lactuca sativa) and chives (Allium fistulosum). These plant species were also grown on earth beds. The vegetables were harvested 30 days after planting, and measurements of the dry and green matter mass were obtained. The growth of tilapia was monitored by obtaining weekly measurements of weight and length. The results showed that fish farming and vegetable cultivation can be combined in a single system, capable of offering good results. Aquaponics proved to be a more advantageous environment for the production of watercress and chives, reaching more than $75 \%$ when compared to earth beds. Tilapia raised in nurseries of this system can achieve a daily growth rate of $0.06 \mathrm{~cm}$ day -1 , provided the correct feeding is done.
\end{abstract}

Key words: Tilapia; Watercress; Chives; Lettuce; Aquaponics.

\footnotetext{
${ }^{1}$ Submetido em 15/12/2019 e aprovado em 27/07/2020;

${ }^{2}$ Secretaria de Ciência e Tecnologia, Cuiabá, MT, Brasil. E-mail: gilvancontini01@ gmail.com - ORICD: https://orcid.org/0000-0003-36502566; lucianobelorodrigues@ gmail.com - ORICD: https://orcid.org/0000-0001-5994-2092; rodrigogobr@ bol.com.br (Autor correspondente) - ORICD: https://orcid.org/0000-0001-9369-8935
}

Agropecuária Técnica, Areia-PB, v. 41, n. 1-2, p. 41-46, 2020

https://doi.org/10.25066/agrotec.v41i1-2.49804 


\section{Introdução}

A aquaponia é uma modalidade de produção de alimentos que envolvem a produção consorciada de peixes e vegetais, num sistema fechado com recirculação de água. O sistema é também considerado sustentável, tendo em vista a oportunidade de recuperar os efluentes da produção de peixe como fertilizante para produção vegetal (Diver, 2006).

A espécie de peixe mais frequentemente utilizada nos sistemas de aquaponia é a tilápia (Oreochromis niloticus). Essa preferência se justifica porque é rústica, tem boa conversão alimentar, tolera altas densidades de estocagem, possui pacote tecnológico de cultivo, variantes melhoradas, alto valor comercial (Marengoni, 2006), crescimento acelerado e reprodução tardia (Kubitza, 2003). Também são criadas outras espécies de peixes, como a carpa comum (Cyprinus carpio), perca gigante (Lates calcarifer), bacalhau australiano do rio (Maccullochella peelii), bagre americano (Ictalurus punctatus), chigã (Micropterus salmoides), truta (Oncorhynchus mykis) e o pacu (Piaractus mesopotamicus) (Rakocy, 2006). Portanto, embora a quantidade de espécies adaptadas à aquaponia não seja numerosa, existem variantes em quantidade suficiente pra atender a preferência dos criadores.

As espécies vegetais adaptadas à hidroponia podem ser recomendadas para o sistema aquapônico. Basicamente, precisam ser espécies vegetais que toleram altos teores de água em suas raízes, e, também, variações nos teores nutricionais (Rakocy, 2007). Já foram produzidas varias espécies de vegetais como, por exemplo, salsinha, menta, alface, agrião, rúcula e manjericão apresentando bons resultados (Herbert \& Herbert, 2009). O agrião (Nasturtium officinale), planta perene e herbácea, é colhido entre 40 e 60 dias após o seu plantio. Pode ser consumida fresca ou após secagem em estufa (Vaz et al., 2006) e se adapta à condições de alagamento e pH alto. A cebolinha (Alliums choenoprasum), planta perene, é resiste tanto ao frio quanto ao calor, podendo ser cultivada em qualquer época do ano (Filgueira, 2000). A colheita inicia-se entre 55 e 60 ou 85 e 100 dias após a semeadura, sendo feita com o corte dos ramos mais desenvolvidos. Após o corte, a planta regenera, perfilhando e gerando uma pequena touceira, sendo possível obter até três cortes em um mês (Makishima, 1993; Filgueira, 2000). A alface (Lactuca sativa), é plantada e consumida por quase todo território brasileiro, não havendo muitos fatores climáticos limitantes (Costa \& Sala, 2005) e no mercado Brasileiro e destaca-se a crespa com $70 \%$ de preferência, seguida pela americana com $15 \%$, a lisa com $10 \%$ e a romana com 5\% (Suinaga et al, 2013). Havendo também cultivares roxa e com sua folhas frisadas (Sousa et al., 2014).

A produção de hortaliças é proveniente de quatro sistemas de produção: cultivo no sistema hidropônico, convencional, orgânico em campo aberto e no solo (Filgueira, 2005). Porém, a demanda de alimentos vem recebendo uma reorientação. Está se popularizando a preocupação com a qualidade do alimento servido à mesa, principalmente a preocupação com uso indiscriminado de agroquímicos. Exemplo disso são as investidas recentes de organizações não governamentais no legislativo, buscando a proibição do herbicida glifosato, indispensável na agricultura moderna.

Com a presente pesquisa objetivou-se investigar o crescimento de tilápias criadas em pequenos viveiros, com reutilização de água, bem como a adaptação de alface, agrião e cebolinha ao sistema de aquaponia.

\section{Material e Métodos}

O experimento foi instalado no distrito de Paranorte, localizado a $140 \mathrm{~km}$ da sede municipal,

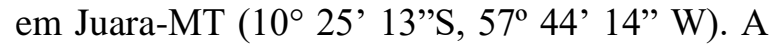
precipitação média anual, registrada nos últimos cinco anos em uma estação pluviométrica local, foi de $2.519,8 \mathrm{~mm}$. No experimento, o sistema de aquaponia foi instalado, basicamente, com criação de peixes em um sistema fechado, composto por viveiro de peixes (caixa d'água de 500 litros). Acima do viveiro, três camas de produção (galões de 50 litros abertos na superfície superior) foram instaladas. As camas de produção foram colocadas sobre um pequeno estaleiro, construído caibros e cavaletes (Figura 1).

Os seguintes materiais foram utilizados: 1 caixa d'água com capacidade de 500 litros; 1 bomba d'água elétrica; 1 luva PVC; 2 conexões T; 3 adaptadores soldável com anel flange; adaptadores com bolsa e rosca; 3 luvas soldável e com rosca; 2 abraçadeiras de nylon, 3 torneiras de 
jardim, 3 flanges; 3 canos PVC (50mm), no comprimento de $28 \mathrm{~cm}$. Os tubos e conexões foram, todos, no diâmetro de $20 \mathrm{~mm}$. Além do material condutor de água, também foram utilizados 4 caibros de ( $1,50 \mathrm{~m}$ cada) e 2 cavaletes (1,20m de altura) para construção do estaleiro. a)

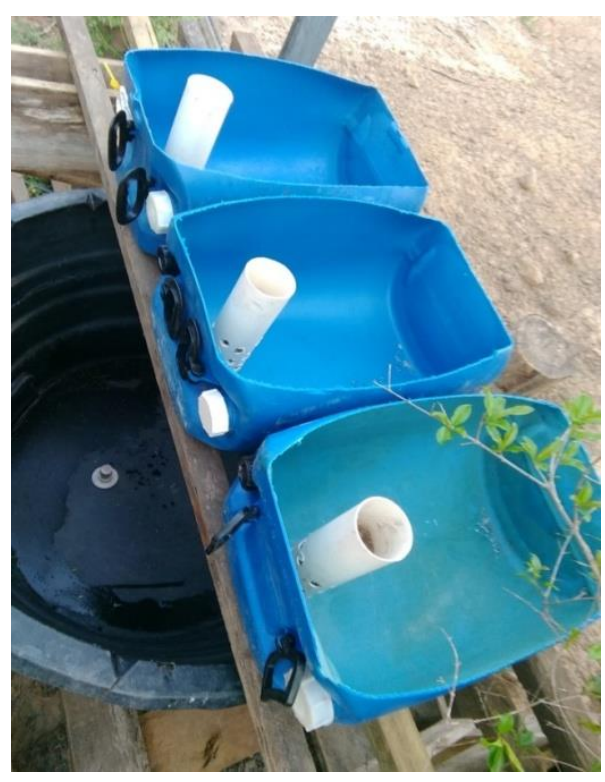

b)

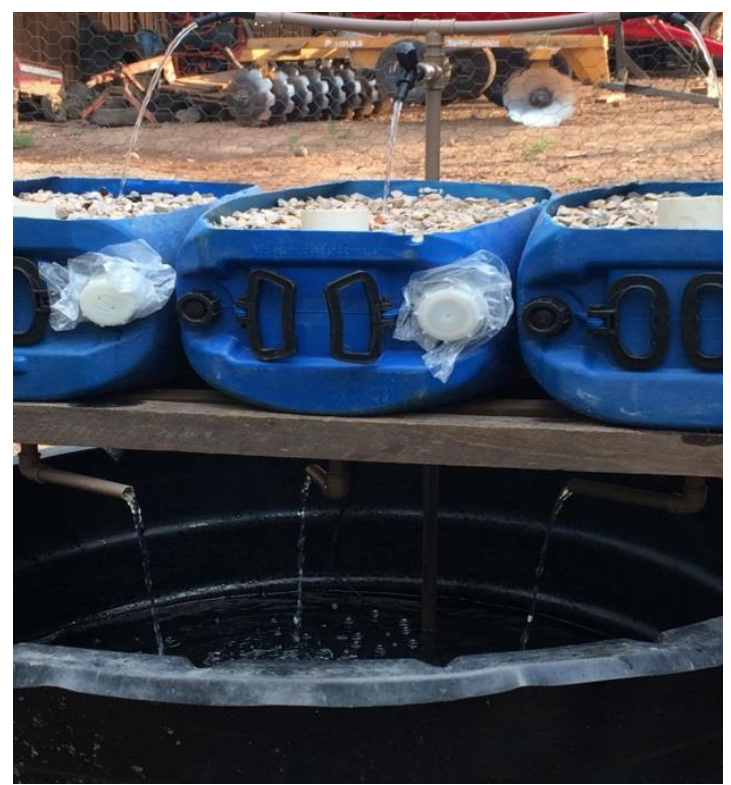

Figura 1 Sistema de aquaponia utilizado com um viveiro de criação de peixes (caixa d'água e três camas de produção). (a) vista superior. (b) vista de perfil.

A água foi conduzida até o sistema por tubulação PVC. Por ser um sistema fechado, com reaproveitamento de água e efluentes, a água precisou ser bombeada da caixa d'água para as camas de produção. Essa circulação forçada foi feita utilizando uma bomba submersa, de funcionamento elétrico, com capacidade de vazão de $1 \mathrm{~m}^{3} \mathrm{~h}^{-1}$. Os canos, dentro das camas de produções, tiveram sua superfície crivada até a metade de sua extensão. As camas de produção foram preenchidas, utilizando a mistura de brita 02 e cacos cerâmicos, na proporção 100:50 (v:v).

$\mathrm{O}$ viveiro de criação dos peixes consistiu em uma caixa d'água, de uso doméstico, com volume máximo de 500 litros. Foram coletados, em uma represa local, 27 alevinos de tilápia (Oreochromisniloticus), com peso médio de $38,5 \mathrm{~g}$. Os alevinos foram alimentados com ração de crescimento extrusada, no tamanho de $4 \mathrm{~mm}$, contendo $36 \%$ de proteína e $500 \mathrm{mg}$ de vitamina C.

As medidas de comprimento, como tamanho dos peixes, foram obtidas com trena métrica. A qualidade da água foi monitorada por meio de medidas de $\mathrm{pH}$ utilizando-se, para isso, um kit de teste de alcalinidade de água. As amostras de água foram avaliadas a cada 7 dias.

A produção vegetal se deu tanto em camas de produção, do sistema aquapônico, quanto em plantio convencional, feito em canteiro de terra. Nas camas de produção, foram utilizados, como material de cultivo, três espécies vegetais cultivadas: agrião, cebolinha e alface. O agrião foi transplantado com altura de $10 \mathrm{~cm}$, numa densidade de 45 plantas $\mathrm{m}^{-2}$. Por sua vez, a cebolinha foi plantada na densidade de 50 plantas $\mathrm{m}^{-2}$. A alface foi transplantada na idade de 30 Dias Após a Emergência (DAE) e densidade de 25 plantas $\mathrm{m}^{-2}$. As mudas foram plantadas diretamente no substrato. Por sua vez, os canteiros foram levantados em solo Podzólico Vermelho-Amarelo, com aplicação de calcário $\left(200 \mathrm{~g} \mathrm{~m}^{-2}\right)$ e adubação com esterco bovino $(5 \mathrm{~kg}$ $\mathrm{m}^{-2}$ ). A densidade de plantio foi a mesma utilizada nas camas de produção.

A massa verde e massa seca foram tomadas sem a separação das raízes da parte aérea das plantas. A massa verde foi obtida tão logo os vegetais foram colhidos. A obtenção dessa variável se justificou por serem consumidas ainda verdes, com as folhas no seu aspecto mais tenro. 
Por sua vez, para obtenção da massa seca, os vegetais foram desidratados ao calor do sol, em lugar ventilado e abrigado contra o acesso de animais. $\mathrm{O}$ aspecto seco do material colhido foi confirmado apenas visualmente.

Todas as medidas envolvendo pesagem, seja de peixe ou de massa vegetal, foram obtidas por meio de uma balança de precisão, com capacidade de $50 \mathrm{~kg}$. Os peixes, por sua vez, foram medidos com auxílio de fita métrica. Por fim, os dados foram submetidos a análise de regressão linear, utilizando o software de análises estatísticas "R".

\section{Resultados e Discussão}

\subsection{Produção Vegetal}

O cultivo de agrião e cebolinha não apresentou sintomas de limitação de crescimento atribuídos ao $\mathrm{pH}$ da água, que, em algumas medidas, apresentou-se próximo a 7,0. Agrião e cebolinha produzem melhor em ambiente com $\mathrm{pH}$ entre 6,0 e 6,8 (Filgueira, 2005). Menciona-se que nenhum fertilizante mineral foi adicionado a água, e que apenas o efluente da produção de peixes foi suficiente para a produção de agrião e cebolinha.

Das espécies vegetais, o agrião foi a que melhor se adaptou. O agrião cultivado em sistema convencional alcança tamanho comercial (10 a 20 $\mathrm{cm})$ por volta de 45 a 50 dias (Filgueira, 2005). Entretanto, o agrião cresceu mais rapidamente no ambiente de aquaponia, atingindo tamanho comercial antes dos 30 dias.

As variáveis massa seca (MS) e massa verde (MV) mostraram-se altamente correlacionadas, conforme estimativa da correlação de Pearson ( $\mathrm{r}$ $=0,97)$. Essa colinearidade já era esperada, uma vez que ambas variáveis são medidas do mesmo material vegetal. Tendo em consideração que agrião, cebolinha e alface são consumidos no seu estado mais tenro, foi dada preferência à análise dos dados relativos à massa verde dos vegetais.

No trabalho, foi possível observar que o agrião é uma espécie de crescimento acelerado, capaz de rapidamente se adaptar ao ambiente e produzir grande volume de biomassa. Foi observado, a partir da segunda semana de cultivo, a emissão de estolões e raízes, de forma a ocupar todos os espaços na cama de produção. Em trinta dias de cultivo, a espécie produziu $1.565 \mathrm{~g} \mathrm{~m}^{-2}$ na aquaponia. No cultivo em canteiro também foi observado o vigor da espécie, que, ao final de 30 dias, produziu $880 \mathrm{~g} \mathrm{~m}^{-2}$ em canteiro de terra (Tabela 1).

Tabela 1 Biomassa produzida nos sistemas aquaponia e em canteiro de terra (convencional)

\begin{tabular}{|c|c|c|c|}
\hline & Sistema & Massa verde & Massa seca \\
\hline \multirow{4}{*}{ Aquaponia } & Cama c/ Agrião & 1565 & 75 \\
\hline & Cama c/ Cebolinha & 75 & 15 \\
\hline & Cama c/ Alface & 55 & 15 \\
\hline & Canteiro c/ Agrião & 880 & 60 \\
\hline \multirow[t]{2}{*}{ Convencional } & Canteiro c/ Cebolinha & 40 & 25 \\
\hline & Canteiro c/ Alface & 360 & 30 \\
\hline
\end{tabular}

$\mathrm{Na}$ literatura relacionada, não foram encontrados estudos comparando o cultivo convencional, em canteiro de terra, com aquaponia. $\mathrm{O}$ crescimento de agrião em hidroponia com soluções nutritivas em diferentes concentrações de nutrientes, vem sendo mais estudada. Ortiz-Hernández et al (2015), por exemplo, mencionam que a espécie produz até 4,37 $\mathrm{g} \mathrm{m}^{-2}$ por planta de massa verde em hidroponia, a depender da solução nutritiva utilizada. No presente estudo, a produção de agrião, observada no sistema de aquaponia, superou em muito a produção reportada por esses autores. Seria necessário cultivá-lo em muito alta densidade 1.372 plantas $\mathrm{m}^{-2}$ para alcançar uma produção de massa verde equivalente.

Por sua vez, a cebolinha também teve rápido crescimento, mas é uma planta cespitosa que cresce emitindo perfilhos. A espécie adaptou-se à cama de produção, produzindo $88 \%$ a mais de biomassa verde, relativamente ao cultivo em canteiro de terra (Tabela 1). Em experimento comparando diferentes soluções nutritivas, Santos et al. (2005) observaram potencial de produção em hidroponia (19 g por planta), 
superando o observado no presente estudo $(8 \mathrm{~g}$ por planta), sugerindo que o efluente da criação de tilápias não fertiliza tão adequadamente a cebolinha quanto uma solução nutritiva cuidadosamente preparada.

De forma geral, o sistema de aquaponia proporcionou maior produção de biomassa, relativamente ao sistema convencional, de cultivo em canteiros de terra. O agrião e a cebolinha adaptaram-se muito bem à aquaponia, produzindo biomassa verde em quantidade superior ao cultivo em canteiro de terra. A biomassa verde (MV) pesou no sistema de aquicultura 88 e $78 \%$ a mais que no sistema convencional para o agrião e para a cebolinha, respectivamente (Tabela 1).

A alface é a principal espécie vegetal cultivada em aquaponia. Nesse sistema, pode produzir plantas pesando entre 40 e $90 \mathrm{~g}$, conforme observado por Castellani et al. (2009). Embora com grande potencial de ser produzido em aquaponia, o cultivo de alface enfrentou condições problemáticas, relativas à incidência de doenças com consequente morte das plântulas. Foi observado sintoma de tombamento, possivelmente causado por Pythium spp. $e$ Rhizoctônia solani. Adicionalmente, menciona- se o acúmulo de resíduos nas camas de produção, provenientes do efluente da criação de peixes. Esses resíduos podem ter formado ambiente propício àqueles agentes fitopatogênicos.

\subsection{Criação de tilápias em viveiro}

O tamanho das tilápias relacionou-se com o tempo de arraçoamento no viveiro através da equação $\hat{y}=12,45+0,06 \times d \quad($ Tabela 2$)$, em que $\hat{y}$ é a estimativa do tamanho dos peixes e "d" é o número de dias. As estimativas geradas pelo modelo demonstram que as tilápias tinham tamanho médio de $12,45 \mathrm{~g}$ no início do experimento, apresentando taxa de crescimento médio de aproximadamente $0,06 \mathrm{~cm} \mathrm{dia}^{-1}$ (ou $0,48 \%$ ao dia), ou equivalente a quase meio centímetro por semana $\left(0,42 \mathrm{~cm}\right.$ semana $\left.{ }^{-1}\right)$. A taxa de crescimento diário foi muito inferior ao observado na literatura relacionada. Menciona-se, por exemplo, taxa de crescimento de $2,42 \%$ ao dia (Carmo et al. 2008). Essas desconformidades podem ser explicadas devido a diferenças importantes, como a linhagem de tilápia estudada bem como a quantidade e qualidade da ração fornecida.

Tabela 2 Tamanho e peso de tilápias amostradas no viveiro de criação. Medidas tomadas em intervalos de uma semana

\begin{tabular}{|c|c|c|c|c|c|c|c|c|c|c|}
\hline \multirow[b]{2}{*}{ Amostra } & \multicolumn{2}{|c|}{$1^{\mathrm{a}}$ semana } & \multicolumn{2}{|c|}{$2^{\mathrm{a}}$ semana } & \multicolumn{2}{|c|}{$3^{\mathrm{a}}$ semana } & \multicolumn{2}{|c|}{$4^{\mathrm{a}}$ semana } & \multicolumn{2}{|c|}{$5^{a}$ semana } \\
\hline & $\begin{array}{l}\text { Tamanho } \\
\text { (cm) }\end{array}$ & $\begin{array}{c}\text { Peso } \\
(\mathrm{g})\end{array}$ & $\begin{array}{l}\text { Tamanho } \\
(\mathrm{cm})\end{array}$ & $\begin{array}{c}\text { Peso } \\
(\mathrm{g})\end{array}$ & $\begin{array}{l}\text { Tamanho } \\
\text { (cm) }\end{array}$ & $\begin{array}{c}\text { Peso } \\
(\mathrm{g})\end{array}$ & $\begin{array}{l}\text { Tamanho } \\
\text { (cm) }\end{array}$ & $\begin{array}{c}\text { Peso } \\
(\mathrm{g})\end{array}$ & $\begin{array}{l}\text { Tamanho } \\
\text { (cm) }\end{array}$ & $\begin{array}{c}\text { Peso } \\
(\mathrm{g})\end{array}$ \\
\hline 1 & 13 & - & 13 & - & 13 & - & 13,5 & - & 15 & - \\
\hline 2 & 13 & - & 12 & - & 12 & - & 13 & - & 16 & - \\
\hline 3 & 13 & - & 13,5 & - & 14 & - & 14 & - & 15 & - \\
\hline 4 & 14 & - & 14,5 & - & 15 & - & 15 & - & 14 & - \\
\hline 5 & 12,5 & - & 13 & - & 13 & - & 13,5 & - & 14 & - \\
\hline 6 & 13 & - & 13 & - & 13 & - & 14 & - & 14 & - \\
\hline 7 & 14 & - & 14 & - & 14 & - & 15 & - & 16,5 & - \\
\hline 8 & 12 & - & 13 & - & 13 & - & 14 & - & 14 & - \\
\hline 9 & 12 & - & 12 & - & 12 & - & 13 & - & 14 & - \\
\hline 10 & 14 & - & 15 & - & 15 & - & 15 & - & 15,5 & - \\
\hline Média & 13,05 & 38,5 & 13,3 & 44,0 & 13,4 & 49,0 & 14,0 & 59,0 & 14,8 & 69,0 \\
\hline & & \multicolumn{2}{|c|}{ Estimativa } & \multicolumn{3}{|c|}{ Desvio Padrão } & \multicolumn{2}{|c|}{ t-valor } & \multicolumn{2}{|c|}{ p-valor } \\
\hline \multicolumn{2}{|c|}{ Interecepto } & \multicolumn{2}{|c|}{12,45} & \multicolumn{3}{|c|}{0,3024} & \multicolumn{2}{|c|}{41,17} & \multicolumn{2}{|c|}{0,0} \\
\hline \multicolumn{2}{|c|}{ Coef. Angular } & \multicolumn{2}{|r|}{0,06} & \multicolumn{3}{|c|}{0,013} & \multicolumn{2}{|c|}{4,60} & \multicolumn{2}{|l|}{0,0} \\
\hline
\end{tabular}

Quanto ao peso das tilápias, não foi possível aplicar qualquer método estatístico de análise. As medidas de peso na Tabela 2 são provenientes de amostra de dez indivíduos. A falta de medidas individuais impossibilitou uma estimativa do erro amostral. Contudo, é possível perceber efeito significativo do arraçoamento sobre o peso médio dos peixes (ganho médio de $1 \mathrm{~g} \mathrm{dia}^{-1}$, ou 2,59\% ao dia). O ganho diário de peso superou em muito o reportado na literatura. Como exemplo, Araújo et al. (2010) mencionam ganho de crescimento diário variando entre $1,58 \%$ a $1,92 \%$. Além dos 
fatores que influenciam a taxa de crescimento já mencionados, é importante destacar que a densidade dos peixes no viveiro também é considerado importante, conforme constatado por esses autores.

\section{Conclusão}

A aquaponia é um ambiente favorável à produção de agrião e de cebolinha, produzindo mais de $75 \%$ que no sistema convencional;

O cultivo de agrião se adapta melhor que os cultivos de cebolinha e alface sistema de aquaponia que no de canteiros em terra, não se recomendando o cultivo de alface;

A criação de peixes e o cultivo de vegetais podem ser conjugados em um sistema de aquaponia com bons resultados;

No sistema aquapônico tilápias crescem a uma taxa de $0,06 \mathrm{~cm}$ por dia.

\section{Referências}

Araújo, W. C Inferências carioevolutivas sobre grupos crípticos de peixes marinhos e estuarinos. 2009. 84 f. Dissertação (Mestrado em Genética e Biologia Molecular) Universidade Federal do Rio Grande do Norte, Natal, 2009.

Costa, C. P.; Sala, F. C. A evolução da alfacicultura brasileira. Horticultura Brasileira, v. 23, n. 1, p. 158-159,2005. https://www.scienceopen.com/document?vid=d213a9c91bd4-4b27-9a49-50cb27b4c887

Carmo, J. L.; Ferreira, D. A. Silva Jr, R. F.; Santos, R. M. S.; Correia, E. S. Crescimento de três linhagens de tilápia sob cultivo semiintensivo em viveiros. Revista Caatinga, v. 21, n. 2, p. 20-26, 2008. https://periodicos.ufersa.edu.br/index.php/caatinga/article $/$ view/645

Castellani, D.; Camargo, A. F. M; Abimorad, E. G. Aquaponics: use of the efluent from the secondary nursey of Macrobrachium amazonicum for the production of hydroponic lettuce (Lactuca sativa) and watercress. Bioikos, v. 23, n. 2, p 67-75, 2009.
Diver, S. Aquaponics - Integration of hydroponics with aquaculture. National Sustainable Agriculture Information Service, 2006.28p.

https://backyardaquaponics.com/Travis/aquaponic.pdf

Filgueira, F.A.R. Novo manual de olericultura: agrotecnologia moderna na produção e comercialização de hortaliças. Viçosa: Ed. UFV, 2000. 402 p.

Filgueira, F. A. R. Novo manual de o olericultura: agrotecnologia moderna na produção e comercialização de hortaliças. 2 . ed. Viçosa: Ed. UFV, 2005. 412 p.

Herbert, S.; Herbert, M. Aquaponics in Australia - The integrations of Aquaculture and Hydroponics.Mudge, Australia, 2008. 140p. https://doi.org/10.18817/repesca.v11i1.1513

Kubitza, F. A evolução da tilapicultura no Brasil: produção e mercados. Panorama da Aquicultura, v. 13, n. 76, p. 25-35, 2003. https://panoramadaaquicultura.com.br

Marengoni, N. G. Produção de tilápia do Nilo Oreochromis niloticus (linhagem chitralada), cultivada em tanques-rede, sob diferentes densidades de estocagem. Archivos de Zootecnia, v. 55, n. 210, p. 127-138. 2006. https://www.redalyc.org/articulo.oa?id=49521001

Makishima, N. O cultivo de hortaliças. Brasília: Embrapa - CNPH: EMBRAPA-SPI, 1993. 116 p. (Coleção plantar, 4).

Ortiz-Hernández, Y. D.; Martínez-Gutiérrez, G. A.; Urrestarazu, M.; Vasquez-Fasquez, L.; Escaminosa-Tinoco, C. Productivity under shade and differrent nutrient solution of Hydroponic watercress (Nasturtium officinale R. BR.). Journal of Plant Nutrition, v. 38, n. $10, \quad$ p. 1495-1504, 2015. https://doi.org/10.1080/01904167.2014.937875

Rakocy, J. E. Ten Guidelines for Aquaponic Systems. Aquaponics Journal, v. 46, 14-17, 2007.

http://blogs.ifas.ufl.edu/santarosaco/files/2013/06/Aquapo nics-Journal-10-Guidelines.pdf

Vaz, A. P. A.; Jorge, M. H. A. Agrião, 2006. Fonte: <http://www.campinas.snt.embrapa.br >. Acesso em 26 fev. 2019 